\title{
MECHANICAL ENERGY TRANSPORT
}

Robert F. Stein

Department of Astronomy and Astrophysics

Michigan State University

East Lansing, MI U.S.A.

and

John W. Leibacher

Space Astronomy Group

Lockheed Palo Alto Research Laboratory

Palo Alto, CA, U.S.A.

\section{INTRODUCTION}

Ladies and Gentlemen, we now reveal to you the secrets of how to create chaos out of order. The existence of a chromosphere or corona requires the existence of motions. A chromosphere or corona requires some non-radiative heat input. There has to be some kind of motion, either oscillatory or quasi-static, to transport the energy up to the chromosphere or corona. This ordered motion may be observed as chaos: microturbulence, macroturbulence, line asymmetries or shifts. of course, it is necessary to actually compute the effects of motions on line profiles in order to see what will really happen.

We review the properties, generation and dissipation mechanisms of three kinds of waves: acoustic, gravity and Alfven waves. These are not the only kinds that can exist, but they will give you some idea of most of the range of wave properties, at least for the low frequency waves for which plasma effects are unimportant. They are pure cases. These different wave modes are distinguished by their different restoring force--pressure for acoustic waves, buoyancy for gravity waves, and magnetic tension for the Alfven waves. Their properties are summarized in Table I. From an observational viewpoint, the most important properties are the relation between temperature and density variations (which change the intensity) and fluid velocity (which shifts the line). Acoustic waves are compressive: In propagating waves the temperature and density vary in phase with the velocity, which is parallel to the energy flux. However, for standing or evanescent waves the temperature and density are $90^{\circ}$ out of phase with the velocity. Gravity waves are slightly compressive: The temperature and density vary oppositely to each other and $90^{\circ}$ out of phase with the velocity, which is parallel to the energy flux. Alfven waves are not compressive: The temperature, density, pressure and the total magnetic field strength remain constant and the motion is transverse to the energy flux. We will come back to these properties in more detail later. 
There are basically two kinds of generation mechanisms: One is direct coupling from the convective motions to the wave motions, either inside the convection zone or penetrating into the photosphere; and the other is thermal overstability. There are only a few basic dissipatiom mechanisms also: Radiation can destroy the restoring force and damp a wave. The other major dissipation processes occur by collisions which diffuse momentum and energy, and produce viscosity, thermal conductivity, and resistivity. Sometimes, instabilities can clump the particles so that collisions occur with a large collective "particle" rather than an individual one. This increases the effective collision rate and enhances the diffusion of momentum and energy. These diffusive transport processes dissipate acoustic waves in shocks, gravity waves in shear layers, and Alfven waves by viscous or Joule heating.

\section{ACOUSTIC WAVES}

\section{A. PROPERTIES}

This material is well-known, so let us quickly run through the basics. As we said, the restoring force for acoustic waves is the pressure. The energy flux is

$$
\mathrm{F}=\mathrm{pu}=\rho \mathrm{u}^{2} \mathrm{v}_{\mathrm{g}}
$$

and the group velocity is

$$
\mathrm{v}_{\mathrm{g}} \simeq \mathrm{s}\left(\mathrm{I}-\mathrm{N}_{\mathrm{ac}}^{2} / \omega^{2}\right)^{\frac{1}{2}}
$$

where $s$ is the sound speed. The acoustic cutoff frequency is

$$
\mathrm{N}_{\mathrm{ac}}=\mathrm{s} / 2 \mathrm{H}=\gamma \mathrm{g} / 2 \mathrm{~s} \text {. }
$$

Acoustic energy propagation can occur only for $\omega>\mathrm{N}_{\text {ac }}(2 \pi / 200 \mathrm{~s}$ for the sun). In the absence of dissipation or refraction the flux must be constant, and the sound speed is roughly constant throughout the photosphere and chromosphere and increases by a factor of 10 going up to the corona, hence the velocity amplitude will scale roughly as

$$
u \propto \rho^{-\frac{1}{2}} \text {. }
$$

Acoustic waves can propagate or be evanescent or standing. The essential difference is that propagating waves transport energy, but evanescent or standing waves don't. In propagating waves with vertical wavelength small compared to the scale height, the pressure, temperature and density vary in phase with the velocity:

$$
\frac{\delta p}{\rho} \simeq \frac{u}{s}, \frac{\delta T}{T} \simeq(\gamma-1) \frac{u}{s}, \frac{\delta p}{p} \simeq \gamma \frac{u}{s} .
$$

In standing or evanescent waves although the pressure, temperature and density fluctuations are of the same order as in propagating waves, they vary 90 degrees out of phase with the velocity

$$
\frac{\delta \rho}{\rho} \simeq-i \frac{u}{s}, \frac{\delta T}{T} \simeq i(\gamma-1) \frac{u}{s}, \frac{\delta p}{p} \simeq-i(2-\gamma) \frac{u}{s} .
$$


Since the energy flux is the average over a period of the pressure times the velocity, the average flux will be zero. Also the vertical phase velocity of evanescent waves will be infinite, so that the motions will be in phase all the way up and down through the atmosphere. Figure 1 shows a portion of the diagnostic diagram that Jacques Beckers showed yesterday, to remind you that the acoustic waves occur in the high frequency region. Later we will come to gravity waves, which occur in the low frequency region. (For more details, see Lighthil1, 1978.)

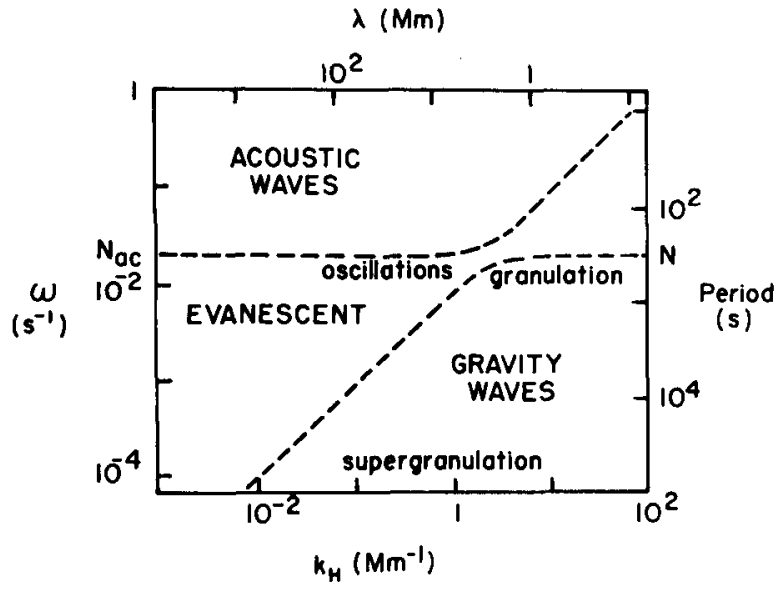

Fig. 1. The k-w diagnostic diagram.

B. GENERATION

1. Direct Generation by Turbulent Motions

Acoustic waves may be generated directly by the turbulent convective motions. This is what is usually called the Lighthill mechanism. The radiated power is roughly the energy density in the turbulent motions divided by the time scale for the turbulent motions, times some efficiency factor. The turbulent energy density is $\rho u^{2}$, the time scale is the eddy to turn over time, which is the length scale of the eddies divided by their velocity, $\tau \simeq \ell / u$, and the efficiency factor is the wave number of the wave times the size of the eddy to some power, $(k \ell)^{2 n+1}$. Thus, the radiated power is

$$
P \simeq \frac{\rho u^{3}}{l}(k \ell)^{2 n+1} .
$$


The exponent $\mathrm{n}$ depends on the kind of emmission: If there is monopole emmission, which corresponds to a mass source, then $n=0$; there is no mass source in the convection zone. If there is dipole emission, which corresponds to a momentum source, which corresponds to an external force, then $\mathrm{n}=1$; in a uniform medium there would be no external force and no dipole emission, but in stars there is an external gravitational field so there is some dipole emission. Finally, for quadruple emission, which corresponds to the action of the Reynolds stresses, $n=2$, and that is the dominant process (Stein, 1967). For acoustic waves:

$$
\mathrm{k} \simeq \omega / \mathrm{s} \text { and } \omega \simeq \tau^{-1} \simeq \mathrm{u} / \ell \text {, }
$$

so

$$
\mathrm{k} \ell \simeq \mathrm{u} / \mathrm{s}=\mathrm{M} \text {, }
$$

the Mach number of the turbulent motions. What you get is the familiar result that the radiated power is proportional to the eighth power of the turbulent velocity:

$$
P \simeq \frac{\rho u^{3}}{l}\left(\frac{u}{s}\right)^{5} \propto u^{8} .
$$

The turbulent velocity that one chooses is very sensitive to the model that one takes for the turbulence, and therefore the emission is very uncertain. But if one makes some crude estimates for the sun,

$$
\rho \sim 10^{-6}, \mathrm{~s} \sim 10^{6}, \mathrm{u} / \mathrm{s} \sim 1 / 4
$$

then

$$
\mathrm{F} \sim \mathrm{Pl} \sim 10^{7} \mathrm{ergs} / \mathrm{cm}^{2} \mathrm{~s} \text {. }
$$

One can also use mixing length theory to see how the flux will depend on stellar properties. From mixing length theory

$$
u \sim\left(\frac{g}{T} \beta\right)^{\frac{1}{2}} \ell
$$

and

$$
\Delta \mathrm{T} \sim B \ell,
$$

where

$$
B=-\left[\mathrm{dT} / \mathrm{d} z-(\mathrm{dT} / \mathrm{dz}){ }_{\mathrm{AD}}\right]
$$

is the superadiabatic temperature gradient. Hence

$$
F \sim \rho c_{p} \Delta T u \sim \rho c_{p}(g / T)^{\frac{1}{2}} B^{3 / 2} \ell^{2},
$$

so

$$
u-\left(\frac{g F l}{p c_{p}^{T}}\right)^{I / 3} \text {. }
$$

From hydrostatic equilibrium

$$
\rho \propto \frac{\mathrm{P}}{\mathrm{T}} \simeq \frac{\mathrm{g}}{\mathrm{KT}} .
$$


Assume

$$
\mathrm{K} \propto \mathrm{P}^{0.7} \mathrm{~T}^{10} \propto \mathrm{g}^{0.41} \mathrm{~T}^{5.88}
$$

then

$$
F \sim \frac{\rho u^{8}}{s^{5}} \propto g^{-1} T_{\text {eff }}{ }^{17} .
$$

This means that the flux decreases very rapidly as you come down the main sequence, and increases rapidly as you go up to the giants and the supergiants. There are some problems with this. Linsky. and co-workers find that the MgII flux is a good measure of the chromospheric emission and they claim that the ratio of the MgII flux to the total flux of the star is independent of $\mathrm{g}$, which is contrary to what the Lighthill mechanism predicts (Basri and Linsky, 1979). Also if you look at the cool main sequence stars you find that the predicted flux is much less than the scaled chromospheric losses. The predicted wave flux may be increased by including effects of molecular hydrogen on the specific heats and the adiabatic gradient. Just how much is not known. Ulmschneider and Bohn are working on that now. But as of the moment there is still over an order of magnitude discrepancy in those results (Schmitz and U1mschneider, 1979.).

The turbulent motions may also directly excite the "five-minute" oscillation. In a steady state the amplitude of a given mode will be determined by the balance between turbulent generation by the Lighthill mechanism and dissipation by turbulent viscosity (Goldreich and Keeley, 1977):

$$
\frac{\rho \mathrm{u}_{\lambda}^{3}}{\lambda}(\mathrm{k} \lambda)^{5}=\nu \mathrm{k}^{2} \varepsilon_{\mathrm{k}},
$$

where

$$
v=\mathbf{u}_{\lambda} \lambda
$$

Hence, the energy density of an oscillation mode $k$ will be

$$
\varepsilon_{\mathrm{k}} \simeq \rho \lambda^{3} \mathrm{u}_{\lambda}^{2} \mathrm{k}^{3}
$$

where $\lambda$ is the size of the eddy whose turnover time equals the oscillation period, $u_{\lambda} / \lambda=\omega_{k}$. For a Kolmogorov turbulence spectrum, where

$$
u_{\lambda}=u_{H}\left(\frac{\lambda}{H}\right)^{1 / 3},
$$

and $\mathrm{H}$ is the scale height, which is assumed to be the size of the largest turbulent eddies which contain most of the turbulent energy, the oscillation mode energy density is

$$
\left.\varepsilon_{\mathrm{k}} \simeq \rho \mathrm{u}_{\mathrm{H}}^{2}\left(\frac{\mathrm{u}_{\mathrm{H}}}{\mathrm{s}}\right)^{11 / 2}\left(\frac{\omega}{\mathrm{N}}\right)_{\mathrm{ac}}\right)^{-5 / 2} .
$$

2. Thermal Overstability

overstability is an oscillating, thermal instability. There are several kinds of thermal instabilities. The one that works for acoustic waves is the $k-$ mechanism or the Eddington valve. If you have an opacity which increases with temperature, then 
when the gas is compressed, it gets hotter, the opacity goes up, it blocks the flow of radiation, so heat accumulates which raises the gas pressure, which means there is more pressure expanding the gas than would be obtained from just compressing the gas, and so it will have a stronger expansion than its compression and the amplitude will increase. When you actually calculate the growth rates as Ando and 0saki (1975) did, you find that they are very slow. The time scale for a mode to grow is about a thousand periods, and that is so long that the turbulent viscosity has a chance to destroy the overstability. On the other hand, as we have seen, the turbulent motion may also directly excite the modes. And since we certainly see them in the sun, we know something is exciting them. It ought to be pointed out that the calculations show that the fundamental mode is stable. It is, however, seen on the sun, although at a somewhat smaller amplitude than the higher modes. If the calculations are right, at least the fundamental mode must be excited by some other mechanism besides thermal overstability. So thermal overstability may or may not work for the five minute oscillation. Some people have proposed a mechanism of Doppler shifted line opacities as a generation mechanism for sound waves in stellar winds.

\section{DISSIPATION}

What about the dissipation of acoustic waves?

1. Radiation

In the first place photons can transfer energy from the hotter to the cooler regions of a wave which will reduce the restoring force and damp the wave. Calculations show that about $90 \%$ of the wave energy of the acoustic waves is removed in the photosphere. Radiative damping also alters the phase of the temperature and density relative to the velocity (Noyes and Leighton, 1963).

\section{Shocks}

The other damping mechanism, of course, is shocks. As the wave propagates its front steepens, and when the thickness of the wave front becomes comparable to the mean free path of the particles, one gets a shock. It should be remarked that a shock has to do with the steepness of the gradient, not with the size of the velocity. You can have a shock where the velocity amplitude is small compared to the sound speed. The distance a wave must travel for a crest to overtake a trough and a shock develop is

$$
\Delta \mathrm{Z}=2 \mathrm{H} \text { In }\left(1+\frac{\lambda}{2 \mathrm{H}} \frac{\mathrm{s}}{\mathrm{u}} \frac{1}{\mathrm{\gamma}+1}\right) .
$$

Short period acoustic waves will dissipate near the temperature minimum, but longer period waves with periods around the acoustic cutoff period (200 sec) will dissipate higher up. And the five minute oscillation which is evanescent doesn' steepen at all until it gets high enough to become nonlinear. The dissipation length is

$$
L \simeq \begin{cases}\lambda /(M-1) & \text { weak shocks } \\ \lambda & \text { strong shocks, }\end{cases}
$$


and the strength of weak shocks varies as

$$
\mathrm{M}=\mathrm{V}_{\text {shock }} / \mathrm{s} \propto \mathrm{p}^{-1 / 4}
$$

(Stein and Schwartz, 1972).

\section{GRAVITY WAVES}

\section{A. PROPERTIES}

In gravity waves the restoring force is buoyancy, which is similar to convection. The different thing about gravity waves is that, while for acoustic waves there is a natural speed, the sound speed, for gravity waves there is a natural frequency, the buoyancy or Brunt-Vaisala frequency at which a blob will oscillate if displaced:

$$
\mathrm{N}=\left(-\frac{\mathrm{g}_{B}}{\mathrm{~T}}\right)^{\frac{1}{2}}
$$

where $\beta$ is the superadiabatic temperature gradient. In an isothermal atmosphere

$$
\mathrm{N} \rightarrow(\gamma-1)^{\frac{1}{2}} \mathrm{~g} / \mathrm{s} \text {. }
$$

Gravity waves only propagate at frequencies less than this natural buoyancy frequency, and the buoyancy frequency is only real and nonzero in convectively stable regions. You cannot have gravity waves propagating in a convectively unstable region. They cannot propagate or be generated inside the convection zone, only by motions in the stable photosphere. Gravity waves propagate energy in a particular direction, which depends on frequency. The cosine of the angle between the flux and the vertical is

$$
\cos \theta=\omega / \mathrm{N} \text {. }
$$

For the sun, for values that are appropriate for the granulation,

$$
\tau_{\text {granulation }} \simeq 10-20 \mathrm{~min} \text { and } \mathrm{N}_{\text {Tmin }} \simeq 0.03=2 \pi / 3 \mathrm{~min},
$$

the direction of gravity wave energy propagation is

$$
\cos \theta \simeq 1 / 5, \quad \theta \simeq 75^{\circ} .
$$

Figure 2 shows what you would see if you looked at a schlieran photograph of gravity waves produced by an oscillating source. The solid lines are the wave crests and the dashed lines are the wave troughs. The lines intersect at the source. Energy is propagating radially outward at the angle theta and the velocity of the fluid is also radial, parallel to the energy flux, but the phase propagates perpendicular to the energy,

$$
\underline{\mathrm{u}} / / \underset{\sim}{\mathrm{F}} \perp \underset{\sim}{\mathrm{k}} \text {. }
$$

You see different waves moving across the fan, while the fan extends out further and further with time as the energy gets out further and further. The group velocity of gravity waves is

$$
\mathrm{v}_{\mathrm{g}}=\frac{\mathrm{N}}{\mathrm{k}} \sin \theta \text {. }
$$




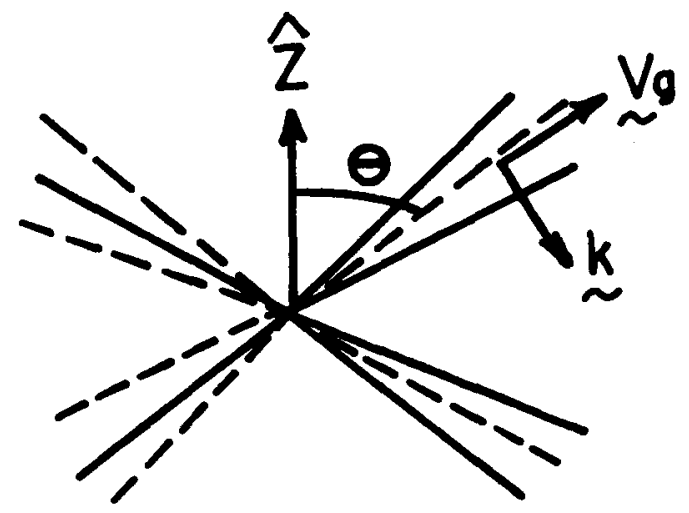

Figure 2: Gravity wave Crests (-) and troughs (--).

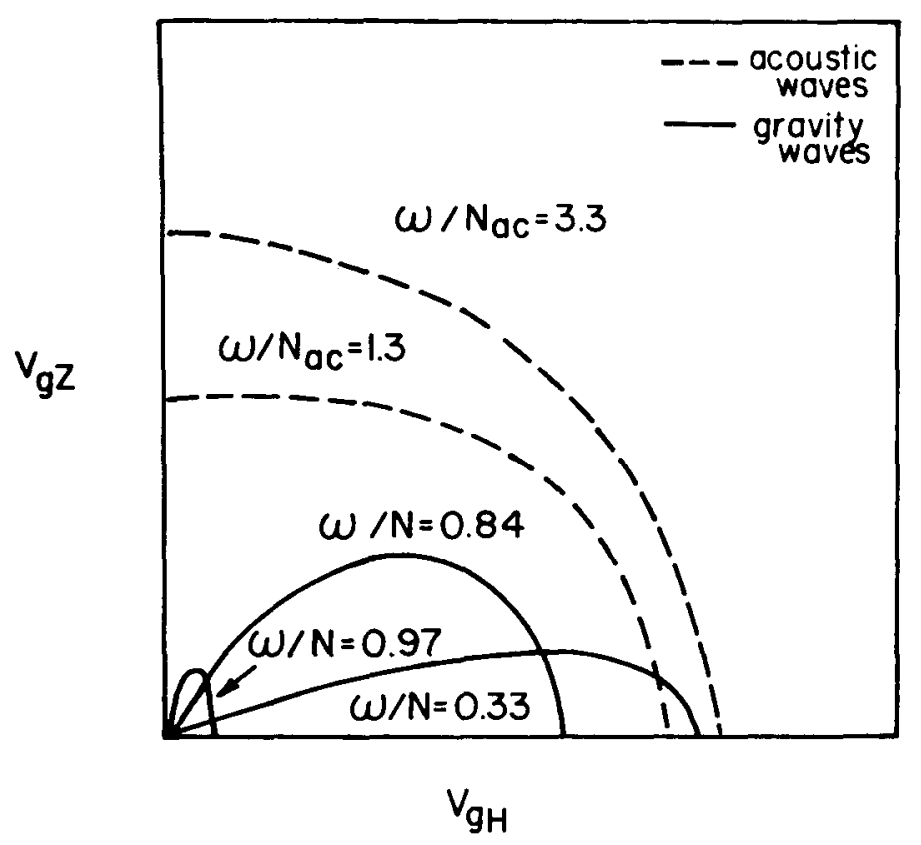

Figure 3: Group Velocity for gravity and acoustic waves. 
For solar granulation $\mathrm{N} / \mathrm{k} \sim 1 \mathrm{~km} / \mathrm{s}$. Figure 3 shows the group velocity for gravity (and acoustic) waves as a function of frequency and direction. Notice that one would observe low frequency, long wavelength waves first. The phase relations between temperature, density, pressure and velocity for low frequency gravity waves are similar to evanescent acoustic waves:

$$
\frac{\delta \rho}{\rho}=i \frac{u}{s}, \frac{\delta T}{T}=-i \frac{u}{s}, \frac{\delta p}{p}=\frac{\omega}{s k_{H}} \frac{u}{s} \ll \frac{u}{s} .
$$

Pressure fluctuations are in phase with velocity but very small, while temperature and density are $90^{\circ}$ out of phase with velocity (Pittway and Hines, 1965; Lighthi11, 1978, Chapter 4).

B. GENERATION

Gravity waves are ubiquitous in the atmosphere of the earth; they are produced by any slow motion. Therefore, they should also be present in the Sun. There are two common generation mechanisms.

\section{Penetrative Convective Motions}

One of the main ways gravity waves will likely be produced is by the penetrative convective motions. One can think of the penetrative convection as blobs pushing on the boundary of the stably stratified layer. The amplitude of the wave produced will be comparable to the amplitude of the penetrative motion,

$$
\left|{\underset{\sim}{\text { wave }}}_{\text {wave }}\right|=\left|\mathrm{u}_{\sim \text { penetration }}\right| \text {. }
$$

This has been verified in laboratory experiments (Townsend, 1966). However, since on1y frequencies that are less than the buoyancy (Brunt-väisälä) frequency can propa-

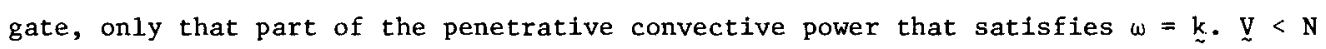
will contribute to the production of gravity waves. This mechanism is similar to the Lighthill mechanism, but with an efficiency near one.

If you make a rough estimate for the solar granulation, taking a velocity of $1 \mathrm{~km} / \mathrm{sec}$ and the appropriate length scales, then

$$
\mathrm{F} \sim \rho \mathrm{u}^{2} \mathrm{v}_{\mathrm{gz}} \sim 3 \times 10^{-7} \times 10^{10} \times \frac{1}{3} 10^{5} \sim 10^{8} \mathrm{erg} / \mathrm{cm}^{2} \mathrm{~s} .
$$

This flux will, however, be greatly reduced by the strong radiative dissipation of gravity waves, which we discuss below.

\section{Shear}

Gravity waves can also be produced by the shear that will arise from the supergranule motions. Supergranule flows have a cellular structure. Conservation of mass requires that a gradient of the vertical momentum flux produces a horizontal momentum flux. Braking is large in the photosphere and produces a large horizontal flow there. Even though the horizontal momentum flux is small in the chromosphere, the chromospheric horizontal velocity is large, because of the small density. The horizontal 
supergranule flow is observed to decrease from $\sim 0.8 \mathrm{~km} / \mathrm{s}$ in the low photosphere to $\sim 0.4 \mathrm{~km} / \mathrm{s}$ in the low chromosphere and then increase to $\sim 3 \mathrm{~km} / \mathrm{s}$ in the mid-chromosphere (November, et al. 1979). Where the size of shear becomes comparable to the buoyancy frequency,

$$
\frac{\mathrm{d} \mathrm{U}_{\mathrm{H}}(\mathrm{z})}{\mathrm{dz}} \geq \min \left[\mathrm{N}, \mathrm{N}^{2} \tau_{\operatorname{coo} 1}\right]
$$

(where $\tau_{\text {cool }}$ is the radiative cooling time) the shear layer becomes unstable and radiates gravity waves. Most of the energy is radiated near

$$
\mathrm{k} \simeq \mathrm{N} / \sqrt{2} \mathrm{U}_{\mathrm{H}},
$$

and the growth times are of order

$$
\gamma \simeq 10^{-1} \mathrm{dU}_{\mathrm{H}} / \mathrm{dz}
$$

(Lindzen, 1974). This mechanism will operate in the low photosphere where the cooling time is short and in the high chromosphere where the shear is large.

\section{DISSIPATION}

How do gravity waves dissipate?

1. Wave Breaking

Gravity waves steepen, but instead of forming a shock front they form a thin shear layer, where the fluid velocity changes direction over a very short distance. When that shear becomes comparable to the buoyancy frequency, $d U / d z \simeq N$, turbulence will develop along that wave front. Small scale motions are produced which dissipate the wave motion and damp the wave. To find the condition on the wave amplitude for breaking to occur, we need to calculate du/dz. Let $u=(u, 0, w)$ where $u$ is the horizontal and $w$ the vertical component of the velocity. For gravity waves the Boussinesq approximation holds, so

$$
\nabla \cdot \underset{\sim}{u}=0
$$

which implies that

$$
\frac{\mathrm{d} w}{\mathrm{dz}}=-i k_{\mathrm{H}} \mathrm{u} \text {. }
$$

The wave equation for gravity waves is

$$
\frac{d^{2} w}{d z^{2}}+\left(\frac{N^{2}}{\omega^{2}}-1\right) k_{H}^{2} w=0,
$$

so

$$
\frac{\mathrm{du}}{\mathrm{d} z}=\frac{\mathrm{i}}{\mathrm{k}_{\mathrm{H}}} \frac{\mathrm{d}^{2} \mathrm{w}}{\mathrm{dz}^{2}}=-\mathrm{i}\left(\frac{\mathrm{N}^{2}}{\omega^{2}}-1\right) \mathrm{k}_{\mathrm{H}} \mathrm{w} .
$$

Hence, the condition for gravity wave breaking is

$$
\mathrm{N}^{-1} \frac{\mathrm{du}}{\mathrm{dz}}=\left(\frac{\mathrm{N}^{2}}{\omega^{2}}-1\right)^{\frac{\mathrm{k}_{\mathrm{H}} \mathrm{w}}{\mathrm{N}}}>1
$$


or

$$
\mathrm{w}>\frac{\mathrm{N}}{\mathrm{k}_{\mathrm{H}}}\left(\frac{\mathrm{N}^{2}}{\omega^{2}}-1\right)^{-1}
$$

\section{Radiative Damping}

As we mentioned, there is very severe radiative damping of the gravity waves. Radiation tends to make a wave isothermal, which destroys the buoyancy restoring force. The radiative cooling rate is

$$
\rightarrow \frac{16 k \sigma \mathrm{T}^{3}}{\rho \mathrm{c}_{\mathrm{V}}} \times\left\{\begin{array}{l}
1-\frac{\pi}{2} \frac{\mathrm{k}}{\mathrm{k}} \text { optically thin } \\
\gamma_{\mathrm{R}}=\tau_{\mathrm{R}}^{-1}=\frac{16 \mathrm{kTT}^{3}}{\rho \mathrm{c}_{\mathrm{v}}}\left(1-\frac{\kappa}{\mathrm{k}} \cot ^{-1} \frac{\mathrm{k}}{\mathrm{k}}\right) \\
\frac{1}{3}\left(\frac{\mathrm{k}}{\mathrm{k}}\right)^{2} \text { optically thick. }
\end{array}\right.
$$

where $k$ is the inverse of the photon mean free path (Spiege1, 1957). The optically thin damping time $\rho \mathrm{c} / 16 \kappa \sigma \mathrm{T}^{3}$, increases rapidly with height and exceeds 10 min above

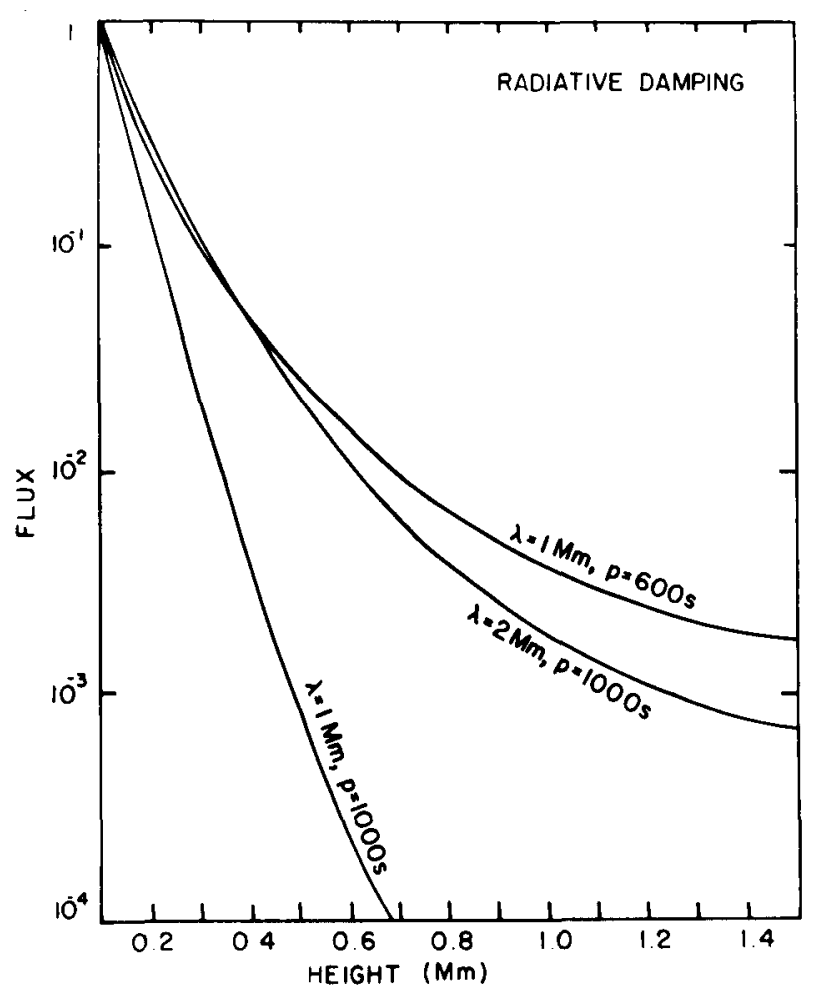

Figure 4. The fraction of flux remaining as a function of height. 
$700 \mathrm{~km}$ above $\tau_{5000}=1$. Damping is severe when the radiative cooling time is shorter than the wave period. The long period, short wavelength waves are the most highly damped. Figure 4, from Barbara Mihalas' thesis (1979), shows the fraction of flux remaining at each height for several wavelengths and periods. In order of magnitude, the flux decreases by $10^{3}$ between the bottom of the photosphere and the mid-chromosphere, for parameters appropriate to granule produced gravity waves. On the other hand, gravity waves generated by penetrative motions a few hundred kilometers above the bottom of the photosphere suffer reduced radiative dissipation and can transmit $2 \%$ of their flux to the chromosphere. Since the radiation which produces the damping of gravity waves also allows for easier penetration of the convection, the wave flux reaching the chromosphere from penetrative convective motions high up in the photosphere is only slightly less than that from the bottom of the photosphere with its severe radiative damping.

\section{Critical Layers}

Gravity waves have one other property which is very different from sound waves: they can interact very strongly with the mean horizontal fluid flow. In particular, they can give the fluid a horizontal acceleration. A layer where the horizontal fluid flow velocity is equal to the horizontal phase speed of the wave, is a "critical layer." Here in the fluid frame, the doppler shifted frequency goes to zero, $\omega-\underset{\sim}{k} \cdot \underline{\sim} \rightarrow 0$, so the waves propagate along the critical layer, $\cos \theta=(\omega-\underset{\sim}{k} \cdot \underset{\sim}{\mathbf{u}}) / \mathrm{N} \rightarrow 0$, and their energy is absorbed. Such a critical layer can arise from the horizontal supergranulation flow or may be produced by absorption of horizontal gravity wave momentum. For the sun, the horizontal gravity wave phase speed is about $1.5 \mathrm{~km} / \mathrm{s}$, which will match the horizontal supergranulation flow somewhere in the mid-chromosphere. The transmission through a critical layer is

$$
T=\frac{F_{\text {trans. }}}{F_{\text {inc. }}}=\exp \left\{-2 \pi\left(R i-\frac{1}{4}\right)^{\frac{1}{2}}\right\} \text {, }
$$

where the Richardson number,

$$
\operatorname{Ri}=\mathrm{N}^{2} /(\mathrm{dU} / \mathrm{dz})^{2}
$$

is the order of 50. Hence, the absorption at critical layers is very large (Booker and Bretherton, 1967; Acheson, 1976). Such critical layers will occur in the chromosphere and will produce localized dissipation of gravity waves there.

The important thing to emphasize about gravity waves is that because they propagate energy mostly horizontally and because of radiative damping, one does not expect too much heating from them. But they are a source of chaos and may contribute substantially to any microturbulence, because they are certain to be there. Their horizontal wavelengths are comparable to granules, $\leq 1 \mathrm{Mm}$, and their vertical wavelengths are only $1 / 4$ as large, which is comparable to the scale height or smaller. 


\section{ALFVEN WAVES}

\section{A. PROPERTIES}

Moving on apace, we now come to Alfven waves. Here the restoring force is magnetic tension. The group velocity is the Alfven velocity,

$$
V_{g}=a=B / \sqrt{4 \pi \rho}
$$

Unlike the sound speed, the Alfven speed increases substantially between the photosphere and the corona, by a factor of $10^{3}$. The flux is

$$
F=\rho u^{2} a=\frac{\delta B^{2}}{4 \pi} a,
$$

and is parallel to the magnetic field. In the absence of dissipation or refraction, the flux remains constant, so the velocity amplitude scales as

$$
u \propto p^{-1 / 4} B^{-1 / 2} \text {. }
$$

The velocity is perpendicular to the magnetic field, i,e. transverse to the direction of energy propagation. Hence Alfven waves act like a vibrating string. There is no compression,

$$
\delta \mathrm{T}=\delta \rho=\delta \rho=0,
$$

so there are no opacity changes. Only Doppler shifts affect the line profiles. The total magnetic field strength, $\left|\mathrm{B}_{\mathrm{O}}+\delta \mathrm{B}\right|$, is constant, so the wave is polarized in part of the arc of a circle, along which the magnetic vector swings back and forth (see e.g., Bazer and Fleischman, 1959; Barnes and Hollweg, 1974).

Most people up until recently have considered uniform magnetic fields. But we know that in the corona the magnetic field is very inhomogeneous. Luckily it turns out that waves in inhomogeneous fields are rather similar to the internal waves in a uniform field. If you consider a thin flux tube surrounded by plasma in a weaker field, several different modes occur (Roberts and Webb, 1978; Wilson, 1979; Wentzel, 1979a). First, there is an axisymmetic mode which is just the torsional Alfven wave, propagating along the flux tube at the Alfve: speed inside the tube, $c=a_{i}$. Second, there is another axisymmetic mode which is like the slow mode, a sound wave propagating along the flux tube with

$$
c^{2}=a_{i}^{2} s_{i}^{2} /\left(a_{i}^{2}+s_{i}^{2}\right) .
$$

It has a short wavelength and its amplitude is concentrated at the surface of the tube. For these waves to excite waves outside the tube, the phase velocity inside the tube would have to be greater than the sound or the Alfven speed outside. In general that is only possible for these modes when the inside density is less than the outside density. Third, there are other modes which are not axisymmetric, and act like vibrating string modes. Their phase velocities are essentially an average of the Alfven speed inside and outside the tube, 


$$
c^{2}=\left(B_{i}^{2}+B_{e}^{2}\right) / 4 \pi\left(\rho_{i}+\rho_{e}\right)
$$

These modes can have a resonance where the phase velocity of the tube mode equals the local Alfven speed. At that resonant point the amplitude of the perpendicular (to the magnetic field) velocity and electric field becomes large. other perturbed quantities are unaffected by the resonance. Thus, even in an inhomogeneous corona the waves are similar, although not identical, to those in a homogeneous corona. The main difference is that for these tube or surface wave modes there exists a resonance at places in the flux tube where $c=a$.

\section{B. GENERATION}

What about Alfven wave generation?

\section{Convective Motions}

Alfven waves can be generated by the convective motions, similar to the Lighthill mechanism for the sound waves. But, because the magnetic field channels the motions, monopole rather than quadrupole emission occurs. The radiated power is

$$
\mathbf{P}=\frac{\rho \mathbf{u}^{3}}{\ell}(\mathrm{k} \ell) \text {. }
$$

For Alven waves,

$$
\mathrm{k}=\frac{\omega}{\mathrm{a}}, \omega=\frac{\mathbf{u}}{\mathrm{l}},
$$

so

$$
\mathrm{k} \ell=\frac{\mathrm{u}}{\mathrm{a}}=\mathrm{M}_{\mathrm{B}} \text {. }
$$

Thus

$$
P=\frac{\rho u^{3}}{l}\left(\frac{u}{a}\right)
$$

(Kulsrud, 1965: Kato 1968). Rough estimates for the sun $\rho \sim 10^{-6}$, u $\sim 10^{5}$, a $\sim 10^{6}$ predict an Alfven wave flux from strong field regions of

$$
\mathrm{F} \sim \mathrm{Pl} \sim 10^{8} \mathrm{erg} / \mathrm{cm}^{2} \mathrm{~s} \text {. }
$$

However, anything which jiggles a magnetic field line will also generage Alfven waves, so granules will generate Alfven waves and supergranules will generate Alfven waves. Granules have larger velocities and so are more important. They produce a flux

$$
F=\rho u^{2} a
$$

For typical granule velocities of $1 \mathrm{~km} / \mathrm{s}$,

$$
F \simeq 3 \times 10^{-7} \times 10^{10} \times 10^{6}=3 \times 10^{9} \mathrm{ergs} / \mathrm{cm}^{2} \mathrm{~s} \text {. }
$$

(See, however, Hollweg, 1979.)

What we are really interested in is the average flux, so we have to include the fact that the flux tubes will spread with height and that the Alfven speed increases with height. The waves produced by granular motions have fairly long wavelength so 
they see the change in Alfven speed roughly as a discontinuity. In this case the transmission coefficient is

$$
T=\frac{4 a_{1} a_{2}}{\left(a_{1}+a_{2}\right)^{2}} \simeq 4 \frac{a_{1}}{a_{2}} \simeq 10^{-3} .
$$

The average flux is

$$
F=F_{0} \frac{A_{\text {photo }}}{A_{\text {corona }}} 4 \frac{a_{\text {photo }}}{a_{\text {corona }}},
$$

where $A$ is the flux tube area. Since the magnetic flux is constant along a $f l u x$ tube, $B A=$ constant, so Aa $\alpha_{\rho}-1 / 2$. Thus, the average flux will be

$$
\begin{aligned}
F & =F_{0}\left({ }^{\rho} \text { corona } / \rho^{\rho} \text { photosphere }\right)^{1 / 2} \\
& \simeq 10^{-4} \mathrm{~F}_{0} \\
& \simeq 3 \times 10^{5} \mathrm{erg} / \mathrm{cm}^{2} \mathrm{~s}
\end{aligned}
$$

which is fairly substantial, enough to heat the corona.

I have not made a distinction between Alfven waves and magnetohydordynamic waves. In a strong field the Alfven and the fast mode are similar and the slow mode is an acoustic wave propagating along the flux tube.

\section{Thermal Overstability}

Alfven waves may also be generated by thermal overstability. This is not the $k$ mechanism, but the Cowling-Spiegel mechanism (Cowling, 1957; Moore and Spiegel, 1966). Here buoyancy acts as a driving force which tends to destabilize the system and make it depart from equilibrium. Magnetic tension acts as a restoring force which tends to bring it back to equilibrium and radiative transfer decreases the destabilizing effect of the buoyancy force. Since there will be less destabilizing effect on the way back than there was on the way out from equilibrium, the magnetic tension will return the system to equilibrium faster than it departed, and the wave amplitude will grow. Because the buoyancy must be destabilizing this mechanism works only in convectively unstable regions. One can calculate the growth rate by equating the rate of working of this buoyancy force with the kinetic energy of the waves (Parker, 1979). The buoyant force is

$$
\mathrm{F}_{\mathrm{B}}=\Delta \rho \mathrm{g}=\rho \mathrm{g} \frac{\Delta \mathrm{T}}{\mathrm{T}} .
$$

The temperature fluctuation is the temperature difference between the adiabatically displaced fluid in the wave and mean temperature at its displaced level, reduced by diffusive radiation cooling:

$$
\Delta \mathrm{T}=A \lambda \beta \frac{t}{\tau_{\text {cool }}},
$$

Where $A$ is the wave amplitude, $\lambda$ is the wave length, $B$ is the superadiabatic temperature gradient, $t$ is the period, and the diffusive radiation cooling time is 


$$
\tau_{c o 01} \simeq \frac{\lambda^{2}}{c l_{m f}} \frac{E_{\text {gas }}}{E_{\text {rad }}}=\frac{k \lambda^{2}}{c} \frac{\rho c_{p} T}{\mathrm{aT}^{4}} \simeq \frac{\rho c_{p}}{k \sigma T^{3}}(\kappa \lambda)^{2},
$$

which is assumed to be much greater than the period. The growth time $\gamma^{-1}$, is the time it takes the buoyant work, $\mathrm{vF}_{B}$, to supply the wave energy $\frac{1}{2} \rho v^{2}$, where $v=A a=A \lambda / t$ :

$$
\gamma^{-1} \mathrm{vF}_{\mathrm{B}}=\frac{1}{2} \rho \mathrm{v}^{2} \text {. }
$$

Thus

$$
\gamma \simeq \frac{g B}{T \omega^{2} \tau_{\operatorname{coo} 1}}=\frac{t_{\text {osc }}^{2}}{t_{\text {eddy }}{ }^{2} \tau_{\operatorname{coo} 1}}
$$

How does this growth rate depend on stellar properties? For an opacity

$$
\kappa=\ell_{\operatorname{mfp}}^{-1} \propto \mathrm{P}^{1.7} \mathrm{~T}^{9}
$$

hydrostatic equilibrium gives

$$
P \simeq \frac{g \rho}{k} \propto g^{\cdot 6_{T}-6},
$$

so

$$
\kappa \propto g / T .
$$

The wave frequency is

$$
\omega^{2} \sim a^{2} / \lambda^{2} \sim B^{2} / p \lambda^{2} \propto B^{2} g-.6 T+7 \lambda^{-2},
$$

and the cooling rate is

$$
\tau_{c \circ 01}^{-1} \simeq \frac{4 \mathrm{~F}}{\rho c_{p} T_{k} \lambda^{2}} \propto g^{-1.6} \mathrm{~T}^{11} \lambda^{-2},
$$

From mixing length theory,

$$
\begin{aligned}
\tau_{\text {eddy }}{ }^{-2} & =\frac{\mathrm{g} \beta}{\mathrm{T}} \simeq\left(\frac{\mathrm{gF}}{\rho \mathrm{C}_{\mathrm{P}} \mathrm{Tl} l^{2}}\right)^{2 / 3} \\
& \propto \mathrm{g}^{1.6} \mathrm{~T}^{5.25},
\end{aligned}
$$

where $\ell=\mathrm{H} \propto \mathrm{T} / \mathrm{g}$. Hence the growth rate varies with stellar gravity, surface temperature, and magnetic field as

$$
\gamma \propto \mathrm{g}^{3 / 5} \mathrm{~T}^{9.25} \mathrm{~B}^{-2} \text {. }
$$

\section{DISSIPATION}

1. Viscous and Joule Heating

How do Alfven waves dissipate? Alfven waves don't steepen and form shocks, because the Alfven speed is independent of wave amplitude, since the magnetic field strength is constant and they are not compressive. They can still dissipate by particle collisions which produce Joule or viscous heating. However, the damping lengths 
are large, although they may be comparable to coronal loop dimensions. The Joule and viscous heating rates are

$$
Q_{J}=n J^{2}=n c^{2} k^{2}(\delta B)^{2} /(4 \pi)^{2}
$$

and

$$
\mathrm{o}_{\mathrm{V}}=\mu \mathrm{k}^{2} \mathrm{u}^{2}
$$

The damping rate is

$$
\gamma=\mathrm{Q} / 2 \mathrm{E},
$$

where

$$
E=\rho u^{2}=\delta B^{2} / 4 \pi,
$$

and the damping length is

$$
\mathbf{L}=\mathbf{a} / \gamma \text {. }
$$

For Joule heating

$$
\begin{aligned}
\mathrm{L}_{\mathrm{J}} & \simeq 8 \pi \mathrm{a}^{3} / \mathrm{nc} \mathrm{c}^{2} \\
& \simeq 10^{20} \mathrm{n}^{-3 / 2} \mathrm{~T}^{3 / 2} \mathrm{~B}^{3} \mathrm{P}^{2} \mathrm{~cm},
\end{aligned}
$$

where the resistivity is

$$
\eta=\frac{\mathrm{m}_{\mathrm{e}}^{\nu} \operatorname{col1}}{\mathrm{ne}^{2}}=\frac{\pi \mathrm{e}^{2} \mathrm{~m}^{1 / 2}}{(\mathrm{kT})^{3 / 2}} \text { In } \Lambda \simeq 10^{-7} \mathrm{~T}^{-3 / 2}
$$

For viscous heating

$$
\begin{aligned}
\mathrm{L}_{\mathrm{V}} & \simeq 2 \rho \mathrm{a}^{3} / \mu \omega^{2} \\
& \simeq 10^{23} \mathrm{n}^{-1 / 2} \mathrm{~T}-5 / 2 \mathrm{~B}^{3} \mathrm{P}^{2} \mathrm{~cm},
\end{aligned}
$$

where the viscosity is

$$
\mu \simeq \frac{1}{3} \mathrm{n} \mathrm{m}_{\mathrm{p}} \mathrm{v}^{2} / \nu_{\mathrm{col} 11} \simeq 10^{-15} \mathrm{~T}^{5 / 2} .
$$

Viscous damping is more important than Joule heating in the corona and visaversa in the photosphere and chromosphere. If the fields are weak, then the Joule dissipation will be significant in the photosphere. Hence Alfven waves can only get through from the photosphere up into the corona in strong field regions. That used to be a serious problem before we knew that fields come in little patches of high field strength. Now it is not. For typical coronal loop parameters $\left(\mathrm{n} \sim 10^{10} \mathrm{~cm}^{-3}, \mathrm{~T} \sim 2 \times 10^{60} \mathrm{~K}, \mathrm{~B} \sim 100 \mathrm{G}\right.$, $\mathrm{L}-10^{10} \mathrm{~cm}$ ) the viscous damping length is

$$
\mathrm{L}_{\mathrm{V}} \sim 10^{8} \mathrm{P}^{2} \mathrm{~cm}
$$

which is comparable to the loop length for short period waves. 
Because the corona is inhomogeneous, the Alfven waves are really tube modes, and have a resonance where the tube phase speed is equal to the local Alfven speed. In this resonant region the wave amplitude is large. Hence large currents and large Joule heating will occur in the narrow resonant layer. The rate of heating is controlled by the rate at which energy can flow into that resonant region, and has been calculated by Ionson (1978) and Wentzel (1979b):

$$
\gamma=\pi \omega k_{r} \Delta r\left(\Delta a^{2} / a^{2}\right)\left(2+\rho_{i} / \rho_{e}+\rho_{e} / \rho_{i}\right)^{-1} .
$$

They thought this was the rate at which waves were radiating energy away. It isn't. It is the rate of energy flow into the resonant region (Hollweg, 1979). There is a problem of how the energy released in this very small resonant region volume is transferred to the rest of the large coronal volume where it is needed. Nobody has figured out how that is done. This is a problem for just about every type of Alfven wave dissipation, except for the Joule and the viscous dissipation. The reason is that in order to rapidly dissipate the Alfven wave energy the currents must be clumped, and so the dissipation occurs in a small region.

2. Mode Coupling

In the presence of inhomogenieties the Alfven wave will couple to other wave modes. Coupling will be large between wave modes whose wave vectors' difference is comparable to the inverse of the inhomogeniety scale length. The coupling ratio between two modes is roughly

$$
|\Delta \mathrm{kL}|^{-1}
$$

where $\Delta \mathrm{k}$ is the difference in $\mathrm{k}$ between the two modes and $\mathrm{L}$ is the length scale of the inhomogeniety (Me1rose, 1977). In a strong field the major coupling occurs between Alfven and fast mode waves that are propagating in the direction of the magnetic field, because both of them are propagating nearly at the Alfven speed, so they will stay together. The difference in wave vector is

$$
\Delta k=\Delta\left(\frac{\omega}{c}\right)=\frac{\omega}{c} \frac{\Delta c}{c},
$$

where $c$ is the phase speed of the mode. For propagation close to the magnetic field direction

$$
\begin{aligned}
& c_{+}^{2}=a^{2}+s^{2} \theta^{2}, \\
& c_{-}^{2}=s^{2}\left(1-\frac{s^{2}}{a^{2}} \theta^{2}\right) . \\
& c_{A}^{2}=a^{2}\left(1-\theta^{2}\right) .
\end{aligned}
$$


So

$$
\begin{aligned}
\frac{\Delta c}{c} & =\frac{c_{+}-c_{A}}{a}=\left(1+\frac{1}{2} \frac{s^{2}}{a^{2}} \theta^{2}\right)-\left(1-\theta^{2} / 2\right) \\
& \simeq \theta^{2} / 2 .
\end{aligned}
$$

and

$$
\Delta k / k \simeq \theta^{2} / 2 \text {. }
$$

Thus the coupling ratio is

$$
\simeq 2(\mathrm{~kL})^{-1} \theta^{-2} \text {. }
$$

When $\theta<\left(\omega / \Omega_{i}\right)^{\frac{1}{2}}$, where $\Omega_{i}$ is the ion-cyclotron frequency, ion cyclotron effects increase $\Delta \mathrm{k}$. So the maximum coupling occurs at this critical angle and is

$$
|\Delta k L|^{-1} \simeq(k L)^{-1}\left(\Omega_{i} / \omega\right)
$$

(Me1rose, 1977). There will not be much coupling if the waves are not propagating along the field direction, nor will there be much coupling between the Alfven and slow modes. The fast mode, if it gets any energy, will form shocks and dissipate, so that this is a round about way in which the Alfven waves can dissipate their energy.

\section{Alfven Wave Decay}

The Alfven waves can also decay. If a set of waves satisfy the resonance condition,

$$
\begin{aligned}
& \omega_{0}=\omega_{1}+\omega_{2}, \\
& \underset{\sim}{k_{0}}={\underset{\sim}{k}}_{1}+k_{\sim},
\end{aligned}
$$

which is essentially energy and momentum conservation, then one wave can decay into two. In this case a forward moving Alfven wave can decay into a backward moving Alfven wave and a slow mode pressure wave, at the rate

$$
\gamma \approx \omega\left(\frac{a}{s}\right) \frac{k}{\Delta k}\left(\frac{\delta B}{B}\right)^{2}
$$

(Kaburaki and Uchida, 1971). For a broad spectrum of incident waves, only those that stay in resonance for a decay time, i.e. have $\Delta k / k \sim \Delta \omega / \omega \leq \gamma / \omega$, can decay. So the decay rate is

$$
\gamma \simeq \omega\left(\frac{a}{s}\right)^{\frac{1}{2}}\left(\frac{\delta B}{B}\right) .
$$

(Sagdeev and Galeev, 1969). There seems to be some disagreement between the calculated decay rates and the fact that one sees the Alfven waves in the solar wind at the earth. 
4. Current Dissipation

Finally, I want to talk a little about current dissipation. Currents are produced not only by Alfven waves, but by any twisting motion of the magnetic flux tubes, for instance a quasi-static twisting motion. Current or magnetic field dissipation is a diffusive process due to single or collective particle collisions. The characteristic resistive diffusion time scale is

$$
\tau_{R}=\frac{2 \mathrm{E}}{\mathrm{Q}_{\mathrm{J}}}=\frac{2 \mathrm{~B}^{2}}{8 \pi n \mathrm{~J}^{2}}=4 \pi \mathrm{L}^{2} / n \mathrm{c}^{2} .
$$

For typical coronal parameters $\tau_{R} \sim 10^{4}$ yrs, too long to be significant. This Joule dissipation time can be reduced either by reducing the width $L$ of the region through which the currents flow or by increasing the resitivity by increasing the effective collision rate. If some instability or resonance filaments the current so the current density is high in a small region, then there can be significant dissipation of the currents. If that occurs and if the current density $\mathrm{J}=\mathrm{n}_{\mathrm{e}}^{\mathrm{ev}} \mathrm{drift}$ becomes large enough so that the drift velocity approaches the electron thermal velocity then substantial numbers of electrons will tend to run away and generate several different types of electrostatic waves. These waves bunch the ions, so that the electrons collide with the electric field of a large collective charge rather than that of a single ion. This scattering of electrons by the waves increases the effective collision rate, the rate of momentum transfer and hence the resistivity. The enhanced resistivity due to electron scattering by plasma waves is called "anomalous resistivity," and since it occurs in conjunction with current filamentation will shorten the resistive diffusion time tremendously (Papadopolous, 1977; Rosner et al, 1978; Hollweg, 1979). Also if the current density becomes large it will develop large shears or gradients in the magnetic field, which will lead to tearing mode instabilities. Parallel currents attract one another and tend to clump. The clumping of current produces a fluid flow that forces the sheared magnetic field into $x$-type neutral points. Filamented currents are produced with small enough length scales so that the classical, Coulomb collision, resistive diffusion time becomes small and the magnetic field can tear and reconnect and the currents can dissipate (Drake and Lee, 1977). This mechanism has been invoked for the violent energy release in $f l a r e s$ (see Spicer \& Brown, 1980). However, shorter wavelength tearing modes distort the field lines more, which produces a greater restoring force, and also have a smaller volume of magnetic energy they can release, so they may produce a more tranquil quasistatic heating appropriate for coronal flux tubes. The tearing instability has a lower threshold than the current driven instabilities which lead to anomalous resistivity. To get significant current dissipation by any mechanism, the dissipation must occur in such small volumes that the transfer of the resulting heat to the rest of the corona is a serious problem. 


\section{CONCLUSION}

In conclusion, there are many kinds of different wave motions in the sun; acoustic, gravity, Alfven waves, and other kinds of magneto-acoustic-gravity wave modes; maybe even higher frequency waves like whistlers. All of these ordered motions may contribute to the chaos observed in stellar atmospheres. In order to develop diagnostics, somebody has to take self-consistent calculations of these waves with the right velocities, temperatures, densities and pressures and calculate the effects on the line profiles of each wave mode.

To summarize the major roles of the waves we have discussed today: Acoustic waves can heat the low chromosphere but not the corona. The evidence is partly observational: the observed nonthermal line widths due to waves in the upper chromosphere are too small, and also theoretical: increasing the driving amplitude at the bottom of the atmosphere only increases the dissipation of the acoustic waves in the chromosphere, but doesn't increase the flux through the transition region to the corona.

For gravity waves the motion is mainly horizontal. Their amplitudes will be comparable to the amplitudes of the penetrative convection (granulation). They may contribute to the observed microturbulent velocities, but they are unlikely to be important in the heating.

Alfven waves will dissipate primarily by highly clumped currents in very small regions, so there is the problem of how to get that energy from the small volume where the dissipation occurs to the larger volume of the corona. The nice thing about Alfven waves is that they are observed in the solar wind, and they seem to be important in providing an energy and momentum input to the wind. Someplace between the photosphere and the earth, where the wind is observed, those Alfven waves must be produced. 
TABLE I.

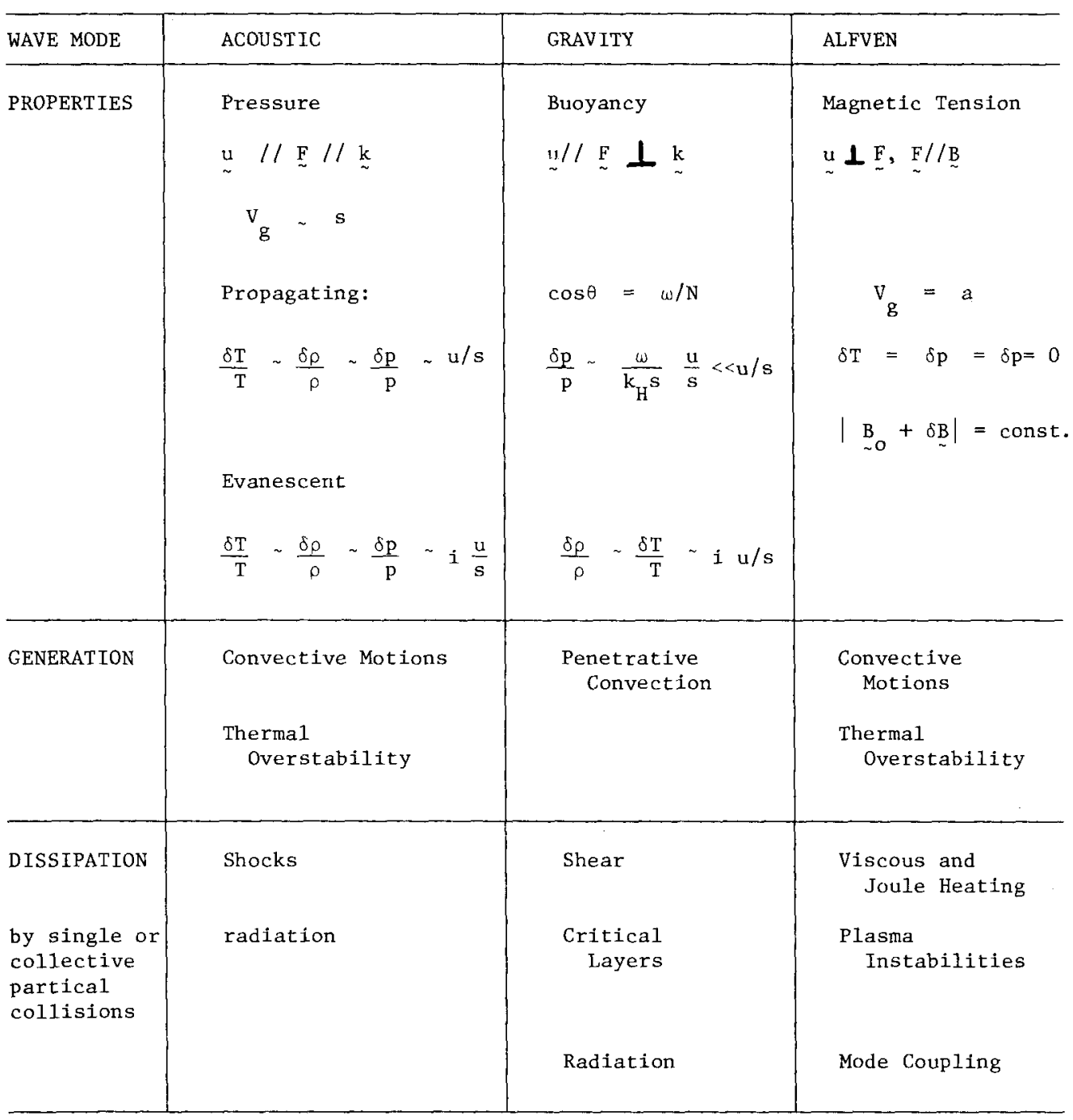


R.F.S. is grateful for support from N.S.F. grant AST-76-22479, NASA grant NSG 7293, and Air Force contract F19678-77-C-0068. J.W.L. is grateful for support from NASA contract NASw-3053 and NAS-5-23758, and the Lockheed Independent Research Fund.

\section{REFERENCES}

Acheson, D. J., 1976, J. Fluid Mech. 77, 433.

Ando, H., and Osaki, Y., 1975, Pub1. Astron. Soc. Jap. 27, 581. Barnes, A., and Hollweg, J. V., 1974, J. Geophys, Res. 79, 2302. Basri, G. S., and Linsky, J. L., 1979, Astrophys. J. (in press). Booker, J. R., and Bretherton, F. P., 1967, J. Fluid Mech. 27, 513. Cowling, T. G., 1957, Magnetohydrodynamics, Interscience, New York. Drake, J. F., and Lee, Y. C., 1977, Phys. Fluids 20, 1341. Goldreich, P., and Keeley, D. A., 1977, Astrophys. J., 212, 243. Hollweg, J. V., 1979, Proc. Skylab Active Region Workshop, ed. F. Q. Orral1, NASA. (in press)

Ionson, J.A., 1978, Astrophys, J., 226, 650 .

Kaburaki, O., and Uchida, Y., 1971, Pub1. Astron., Soc. Jap. 23, 405.

Kato, S., 1968, Pub1. Astron. Soc. Jap. 20, 59.

Kulsrud, R., 1965, Astrophys. J., $121,4 \overline{61}$.

Lighthill, J., 1978, Waves in Fluids, Cambridge University Press, Cambridge, England. Lindzen, R. S., 1974, J. Atm. Sci. 31, 1507.

Me1rose, D. B., 1977, Aust. J. Phys. 30, 495.

Mihalas, B., 1979, Thesis, University of Colorado.

Moore, D. W., and Spiege1, E.A., 1966, Astrophys. J., 143, 871.

Noyes, R. W., and Leighton, R. B., 1963, Astrophys. J., 138, 631.

November, L. J., Toomre, J., Gebbie, K. B., and Simon, G. W., 1979, Astrophys, J., 227,600 .

Papadopoulos, K., 1977, Rev. Geophy. Space Phys. 15, 113.

Parker, E. N., 1979, private communication.

Phillips, 0.M., 1966, The Dynamics of the Upper Ocean, Cambridge University Press, Cambridge, England.

Pitteway, M. L. V., and Hines, C. O., 1965, Can. J. Phys. 43, 2222.

Roberts, B., and Webb, A. R., 1978, Solar Phys. 56, 5.

Rosner, R., Golub, L., Coppi, B., and Vaiana, G. S., 1978, Astrophys. J., $222,317$.

Sagdeev, R. Z., and Galeev, A. A., 1969, Plasma Physics, Benjamin, N. Y.

Schmitz, F., and U1mschneider, P., 1979, Astron. and Astrophys. (in press).

Spicer, D. S., and Brown J. C., 1980, The Sun as a Star, NASA/CNRS (in press).

Spiege1, E. A., 1957, Astrophys. J., 126, 202.

Stein, R. F., 1967, Solar Phys. 2, 385.

Stein, R. F. and Schwartz, R. A., 1977, Astrophys. J., 177, 807 .

Townsend, A. A., 1966, J. Fluid Mech. 24, 307.

Wentze1, D. G., 1979, Astron. and Astrophys. 76, 20.

$1979 \mathrm{~b}$, Astrophys. J. (in press).

Wilson, P. R., 1979, Astron. and Astrophys. 71, 9. 